\title{
Cardiovascular imaging 2010 in the International Journal of Cardiovascular Imaging
}

\author{
Ricardo A. Costa · Johan H. C. Reiber · Frank J. Rybicki • \\ Paul Schoenhagen · Arthur A. Stillman • Johan de Sutter • \\ Nico R. L. van de Veire $\cdot$ Ernst E. van der Wall
}

Received: 7 February 2011/Accepted: 8 February 2011/Published online: 24 February 2011

(C) The Author(s) 2011. This article is published with open access at Springerlink.com

\section{Introduction}

In the year 2010, the International Journal of Cardiovascular Imaging received very interesting contributions dealing with a wide variety of imaging modalities. Papers came from all corners of the world and dealt with both experimental and clinical observations. Since we deal today with image-guided diagnoses, therapies and interventions, there is a growing interest in the increasing capabilities of the existing imaging modalities. Developments in

R. A. Costa

Instituto Dante Pazzanese de Cardiologia, São Paulo, Brazil

J. H. C. Reiber $(\square)$ · E. E. van der Wall

Leiden University Medical Center, Albinusdreef 2,

2333 ZA, Leiden, The Netherlands

e-mail: J.H.C.Reiber@lumc.nl

F. J. Rybicki

Harvard Medical School, Boston, MA, USA

P. Schoenhagen

The Cleveland Clinic Foundation, Cleveland, OH, USA

A. A. Stillman

Emory University Hospital, Atlanta, GA, USA

J. de Sutter · N. R. L. van de Veire

AZ Maria Middelares Gent and Free University Brussels, Brussels, Belgium imaging are therefore not only interesting to imaging experts themselves, but in particular to medical specialists such as cardiologists, radiologists, nuclear medicine specialists, and internal medicine specialists. Improvements in imaging capabilities may directly lead to improved health care. Within the whole spectrum from primary prevention to intervention, myocardial imaging becomes an indispensible tool. Both patients with suspected and known cardiovascular disease may benefit from the tremendous achievements in cardiac and vascular imaging.

Based on these observations, it was thought appropriate to highlight the most valuable papers that were published in 2010 in our journal. The 5 well-known areas were addressed i.e. X-ray angiography, echocardiography, nuclear imaging, magnetic resonance imaging, and computed tomography. These imaging fields were covered by experts in the field and contain a fine selection of noteworthy publications. These contributions may change the landscape of myocardial imaging and potentially influence clinical practice. We hope the reader will enjoy reading the impressive advances in cardiovascular imaging.

\section{X-ray angiography}

A number of very interesting developments in the field of X-ray imaging were published in the Journal over the year 2010 . 
First of all, Janssen et al. [1] presented new approaches for the assessment of the arterial and reference diameters in two-dimensional cardiovascular X-ray images, as well as two new bifurcation models allowing a further increase in accuracy and precision of these QCA techniques [1]. Next, Tu et al. [2] demonstrated that a new 3D QCA approach based on reconstruction from two biplane angiographic views of a phantom allows a highly accurate and precise assessment of obstruction length (difference with true lengths: $0.04 \pm 0.25 \mathrm{~mm}, P<0.01)$ and optimal viewing angle (difference $-1.5^{\circ} \pm 3.6^{\circ}$, $P<0.01)$. The approach corrects for any possible isocenter offset. The team of John Carroll sought to identify optimal viewing regions for coronary segments that provide optimal diagnostic value in terms of minimizing vessel foreshortening and overlap [3]. Based on orthogonal 2D images of the coronary tree of 137 patients, they created a universal optimal view map (OVM) for each coronary segment. Foreshortening values of around $5.7 \pm 3.8 \%$ were obtained.

A $3 \mathrm{D}$ reconstruction approach of the coronary tree from multiple ECG-gated views of rotational X-ray angiography was presented by Liao et al. [4]. Typically, 4-5 views are used for the reconstruction, which takes about $12 \mathrm{~s}$ on a $2 \mathrm{GHz}$ computer. No data on coronary segmentation were reported. Another C-arm based application was described by Hetterich et al. [5]. In this case the C-arm system performs multiple runs around the patient while the ECG is monitored. This approach was directed at visualizing the $3 \mathrm{D}$ anatomy of the coronary system for improved guidance during complex interventional procedures [5].

Van Tongeren et al. [6] studied the usefulness of digital subtraction angiography (DSA) in the evaluation of therapeutic angiogenesis in patients with severe peripheral arterial disease They concluded that better imaging techniques of the collateral circulation are required to assess the efficacy of cell-based therapy.

\section{Echocardiography}

Echocardiography remains the most widely used imaging modality within cardiology and is being used for a variety of cardiac conditions including congenital heart disease, coronary artery disease, cardiomyopathies, valvular diseases and rhythm disorders. The
International Journal of Cardiovascular Imaging received this year several interesting contributions dedicated to conventional echocardiography but also to emerging ultrasound techniques including stress echocardiography, tissue Doppler imaging, strain analysis and three-dimensional echocardiography.

\section{Conventional echocardiography}

Conventional echocardiographic methods have a pivotal role in daily clinical practice and new research expands this role even further. Yuce et al. [7] demonstrated in 112 heart failure patients that a dilated coronary sinus, as measured with echocardiography, is possibly part of the entire process of cardiac remodeling and echocardiographic assessment of a dilated coronary sinus may provide useful additional information, predicting the severity of chronic heart failure and poor functional class. Chockalingam et al. [8] reviewed the clinical presentation of Takotsubo stress cardiomyopathy and proposed a unique diagnostic algorithm for cardiologists acutely managing this cardiac emergency. The pivotal role of conventional echocardiography was emphasized and the nuances of this peculiar acute cardiomyopathy from an echocardiographers' perspective were put forward [9].

Arrhythmias occur frequently in symptomatic patients with mitral valve prolapse, the proper mechanisms of which have not been fully investigated [10]. Turker et al. [11] determined the clinical, echocardiographic, heart rate variability parameters and plasma concentrations of electrolytes and inflammatory markers in predicting the occurrence of ventricular arrhythmias in 58 patients with mitral valve prolapse. Interestingly multivariable logistic regression analysis showed that occurrence of moderate to severe mitral regurgitation, as evaluated by conventional echocardiography, was the only independent predictor of ventricular arrhythmias.

\section{Tissue Doppler Imaging}

Tissue Doppler Imaging (TDI) is used increasingly to detect subclinical disease, to select patients that are eligible for certain treatments or to evaluate the effects of therapeutic interventions. In patients with arterial hypertension, longitudinal myocardial 
function may be impaired while systolic function is still normal. Bilge et al. [12] investigated the relationship between longitudinal myocardial function (by measuring septal and lateral TDI derived strain and strain rate) in 57 patients with untreated stage I hypertension and 48 matched healthy controls. In hypertensive patients longitudinal myocardial function was impaired and related with end organ damage (microalbuminuria, retinopathy) while global circumferential function was still preserved. Betathalassemia major can be associated with myocardial iron deposition causing myocardial dysfunction. Aypar et al. [13] analyzed the efficacy of pulsedwave TDI in predicting iron load in patients with beta-thalassemia major using $\mathrm{T} 2 *$ magnetic resonance as the gold-standard non-invasive test. Pulsedwave TDI was found both sensitive and specific in predicting the presence of myocardial iron load in beta-thalassemia major patients with normal left ventricular global systolic function. The authors concluded that the technique can be used for screening purposes. During percutaneous coronary interventions for acute myocardial infarction, embolization of atherothrombotic material may occur spontaneously or due to mechanic crushing [14]. Distal protection devices can be used during the procedure and allows recovery of any liberated plaque by aspiration before restoration of antegrade flow. Duan et al. [15] assessed the effects of distal protection devices on global and regional systolic function and diastolic function by TDI in 69 patients with anterior infarction, who were randomized to either percutaneous coronary intervention with or without distal protection device. At 3- and 6- month follow-up, the distal protection group showed a higher left ventricular ejection fraction than the control group. Moreover, peak systolic and early diastolic mitral annular velocities obtained by TDI were significantly higher in the distal protection group than in the control group. Also, systolic and diastolic regional myocardial velocities were significantly improved in the distal protection group compared to control group. The past decade, recent research and advanced echocardiographic modalities have provided new insights on proper patient selection, lead implantation, optimization and reasons for non-response in patients treated with cardiac resynchronization therapy. Pavlopoulos and Nihoyannopolous [16] gave a comprehensive review of the selection criteria used to select patients for cardiac resynchronization therapy. The accompanying editorial by Baur [17] critically comments on this controversial issue.

Two- or three-dimensional strain (speckle tracking) and strain rate imaging

Therapeutic decisions regarding operative interventions in patients with mitral stenosis are highly dependent on the presence of symptoms. However, in patients with mild to moderate mitral stenosis, left ventricular systolic function can be affected, even with an apparently normal left ventricular ejection fraction. Ozdemir et al. [18] performed longitudinal two-dimensional strain analysis in 60 patients with mild to moderate mitral stenosis and in 52 healthy controls. Despite normal left ventricular systolic function, mean global longitudinal strain and strain rate were significantly reduced in patients with isolated mitral stenosis. Two-dimensional strain can also be applied to the left atrium. Valocik et al. [19] evaluated the feasibility of velocity vector imaging in the assessment of left atrial volumes and left atrial ejection fraction. A total of 100 patients were evaluated by velocity vector imaging and compared with conventional two-dimensional data. The authors concluded that velocity vector imaging is a feasible method for the assessment of left atrial volumes and left ventricular ejection fraction. It provides close agreement with that measured by conventional twodimensional Simpson's biplane method with significant time saved. Three-dimensional speckle tracking technology is able to perform myocardium deformation and displacement analysis integrating the longitudinal, circumferential and radial components. $\mathrm{Li}$ et al. [20] applied this technology in 15 patients to evaluate the synchrony of myocardial mechanics. They report on a new parameter for the detection of dyssynchrony that might be used to select cardiac resynchronization therapy (CRT) candidates or optimize the device.

Stress echocardiography

Patient safety is an important issue. Today, concerns have risen about the risk of repeated exposure to ionizing radiation. Patients with diabetes are frequently subjected to nuclear scans or non-invasive 
coronary artery CT scans to detect obstructive coronary artery disease. However, dobutamine stress echocardiography is a safe and effective alternative to nuclear imaging and CT. Moreover dobutamine stress echocardiography provides valuable prognostic information. Innocenti et al. [21] studied 322 type II diabetic patients who underwent dobutamine stress echocardiography and followed them for hard endpoints. The presence of viability and severe ischemia were independently associated with higher occurrence of hard cardiac events.

Three-dimensional echocardiography

Both transthoracic and transoesophageal threedimensional echocardiography is increasingly used in clinical practice. Anwar et al. [22] demonstrated in 45 patients that real-time three-dimensional echocardiography was comparable for the assessment of intracardiac thrombi but also provided more detailed information beyond the scope of established twodimensional echocardiography such as thrombus mobility, differentiation between thrombus and myocardium and delineation of changes in thrombi structure. Virtual endoscopy can simulate the tracks of a conventional endoscope using three-dimensional image datasets, such as those from computed tomography or magnetic resonance imaging. Few studies have dealt with echocardiography data. At the University of Shangai researchers have developed a virtual endoscopic system called three-dimensional echocardiographic intracardiac endoscopic simulation system. This system assists with the interpretation of three- dimensional data for heart volume and facilitates the development of three-dimensional heart models. Xue et al. [23] analyzed the feasibility and accuracy (as compared to two-dimensional echocardiography) of three-dimensional echocardiographic virtual endoscopy in the evaluation of congenital heart disease in 40 children. The authors demonstrated that this technique provides intuitive, reproducible and clinically useful visualizations. As a limitation, three-dimensional reconstruction of virtual endoscopy depends on the quality of original images, the presence of artifacts throughout the cardiac cycle. Virtual reality expands the capabilities of noninvasive cardiology and may open new doors for the evaluation of congenital heart disease. Real time transesophageal three-dimensional echocardiography has incremental value as compared to two-dimensional echocardiography for the diagnosis of difficult valvular problems. Paul and Minocha [24] showed in a case report the lenience of identifying thrombosis of a prosthetic heart valve using this technology. One of the most appropriate locations for the use of threedimensional transesophageal echocardiography is the cardiothoracic operating theatre. Gripari et al. [25] demonstrated the routine use of this technique in 100 patients who underwent cardiac surgery. They concluded that imaging with this new probe facilitates intraoperative evaluation of several surgical procedures with an additional clinical value in selected cases.

Ultrasonic intregrated backscatter analysis

This overview of interesting publications on cardiac ultrasound is concluded by the revival of an old ultrasound technique: integrated backscatter analysis. Zhu et al. [26] evaluated whether integrated backscatter is measurable and quantifiable in left atrial posterior walls in 26 patients with chronic atrial fibrillation. From these preliminary data the authors concluded that integrated backscatter of the left atrial posterior wall indexed by pericardium provides an objective quantitative measure of atrial fibrosis. This could be of potential value in patients scheduled for pulmonary vein isolation buts needs further study.

\section{Nuclear cardiology}

In 2010, several excellent papers were published in the field of nuclear cardiology regarding basic/ preclinical research, diagnostic aspects as well as therapy guiding in patients with (suspected) coronary artery disease (CAD) or heart failure. In this overview we highlight some of these papers.

\section{A. Basic/preclinical research}

\section{Imaging of vulnerable plaques}

The presence of activated macrophages is an important predictor of atherosclerotic plaque rupture. Davies et al. [27] evaluated the accuracy of 18Ffluorodeoxyglucese (FDG) microPET imaging for quantifying aortic macrophage content in a rabbit 
model of atherosclerosis. They used 3 groups of rabbits: a control group, a group post aortic balloon injury with 6 months high-cholesterol diet and a group post aortic balloon injury with 3 months highcholesterol diet followed by 3 months of statin therapy. Macrophage density was measured by histological quantification of the atherosclerotic aortas and then compared with in vivo and ex vivo FDG microPET. There was a very strong correlation across all groups between macrophage density and FDG PET uptake derived from ex vivo microPET $(\mathrm{r}=0.95, \quad P<0.01)$. However, the correlation between in vivo FDG PET uptake and macrophage density was insignificant. This study confirms previous findings that differentiation of highly inflamed atherosclerotic aortas from stabilized atherosclerotic aortas might be possible in animal models. Clearly, FDG-PET remains promising for plaque imaging in animal models, but ex vivo results apparently do not always translate into promising in vivo results.

\section{Imaging of cardiac metabolism}

In recent years, PET has emerged as suitable imaging modality to study the metabolic mechanisms of left ventricular dyssynchrony and the effects of cardiac resynchronization therapy. Several PET studies in chronic CRT patients have shown that CRT improves oxygen metabolism and cardiac efficiency [28]. Kitaizumi et al. now evaluated 20 patients very early (1 week) after CRT [29]. All patients underwent 11C-acetate PET and oxygen consumption was measured by the mono-exponential clearance rate of 11C-acetate (Kmono), while the biventricular pacemaker was off and then on. Cardiac efficiency was determined using the work metabolic index which was calculated as (stroke volume index) $\times$ (systolic blood pressure) $\times$ (heart rate)/Kmono. In total 14 patients showed improved cardiac efficiency during biventricular pacing. Importantly, these patients showed decreased oxygen consumption (indicating improved oxidative metabolism) with no changes in hemodynamic parameters. During the 1 year followup cardiac event rate was lower than in the patients without improvement in cardiac efficiency. Although the total number of patients was limited and no difference could be made between ischemic and nonischemic patients, the results of this study indicate that the decrease in oxygen consumption assessed by
PET in the early period after CRT could be a potential marker for improvement of cardiac function and a better outcome. This also illustrates the potential role of PET imaging to evaluate cardiac metabolism in heart failure patients.

\section{B. Diagnosis making in patients with suspected CAD}

Gated SPECT imaging in patients with acute chest pain

Gated myocardial SPECT imaging has become an established very useful technique to improve the specificity of myocardial perfusion imaging. It can help to discriminate true perfusion defects from artifacts and disturbances of wall motion and/or thickening in normally perfused areas may indicate areas of myocardial stunning [30]. Neill et al. [31] nicely underscored the importance of gated SPECT imaging in the early phase of myocardial infarction. They evaluated 68 patients with chest pain at rest and ECG abnormalities in the inferolateral region. All patients were evaluated with gated Tc99-sestamibi SPECT at rest within $24 \mathrm{~h}$ of onset of chest symptoms and myocardial images were semi-quantitatively evaluated for perfusion, motion and thickening. Importantly, of the normally perfused segments $21 \%$ had a motion abnormality and $19 \%$ a thickening abnormality, indicating myocardial stunning in these segments. It was concluded that adding gating results to perfusion results improves the diagnostic accuracy in patients with acute chest patients, especially in the presence of inferolaterall wall ischemia.

\section{Myocardial perfusion imaging as gate keeper to coronary angiography}

Myocardial perfusion SPECT has been promoted for a long time as a gate keeper to coronary angiography. Muzzarelli et al. [32] evaluated a large population of 955 consecutive patients referred to exercise testing and myocardial perfusion imaging. The Duke score (obtained from exercise testing) and the summed difference score (SDS, obtained from perfusion imaging) was evaluated in all patients. According to the guidelines and the available literature, 3 different algorithms for risk stratification were retrospectively applied: (1) exercise testing based risk stratification 
and coronary angiography in intermediate or high risk Duke-score, (2) perfusion imaging based risk stratification and coronary angiography if SDS $\geq 8$ ) and (3) a combination of both with exercise testing as first step and perfusion imaging in case of intermediate risk Duke-score. Coronary angiography would have been suggested in this last scenario in patients with either high risk Duke-score or SDS $\geq 8$ in patients with intermediate risk Duke-score. Strikingly the referral for coronary angiography was $27 \%$ according to the exercise test alone, $13 \%$ using myocardial perfusion imaging and $12 \%$ applying the combined risk stratification. The cost of the diagnostic work-up including coronary angiography were $615 €, 1299 €$ and $598 €$ per patient, respectively. Clearly, the results of this study underline the advantage of a risk based approach applying stress imaging in patients with intermediate risk Duke-score during exercise testing.

\section{Therapy guiding in patients with CAD}

\section{Myocardial perfusion imaging versus fractional flow reserve}

Fractional flow reserve (FFR) measurements have become an important tool in the cathlab to determine if vessels with an intermediate stenosis should be treated invasively. Forster et al. [33] evaluated the accuracy of myocardial SPECT imaging for the detection and allocation of vessel specific perfusion defects in patients with multivessel disease. They used standard distribution territories on SPECT imaging and compared this to FFR measurements in 93 vessels with intermediate stenosis in 72 patients with multivessel disease. MPI detected significant stenosis with a specificity of $85 \%$. However, vesselbased evaluation showed a sensitivity of only $62 \%$ with a specificity of $90 \%$, PPV of $62 \%$ and NPV of $90 \%$. In total $41 \%$ of false negative and false positive myocardial perfusion imaging findings resulted from incorrect allocation of reversible perfusion defects to their determining supplying vessel. In an accompanying editorial by Rees [34] the relative place of SPECT versus FFR measurements is further discussed. Myocardial perfusion imaging will continue to be an important tool to determine which patients should be referred for diagnostic angiography and intervention. However, once the patient is in the catheterization laboratory, is has become clear from this and other studies that quantitative coronary angiography combined with FFR is the method of choice to guide interventions in patients with multivessel disease.

\section{PET viability imaging in ischemic cardiomyopathy}

In patients with CAD and a reduced LV function, coronary artery bypass grafting (CABG) is an important therapeutic option that is, however, still associated with a high perioperative mortality. Viability assessment with different techniques including FDG-PET imaging has been shown to add valuable information for decision making in this clinical setting. However, the exact role of viability assessment to determine suitability for revascularization in these patients has not been defined yet. Boehm et al. [35] studied a large group of 476 patients with CAD and $\mathrm{EF} \leq 35 \%$ who were considered candidates for CABG. A standard care group of 298 patients underwent CABG. In a second FDG-PET-guided management group of 178 patients, 152 underwent CABG and 26 patients were excluded from CABG because of lack of viability. The most important findings were: (1) long-term (up to 10 years) survival was better in the PET-CABG group than in the standard care group, (2) the survival benefit was already present at 30 days follow-up (1.3 vs. $10.3 \%$ mortality); and (3) the criterion of scar tissue alone was not sufficient for the selection process, also other viability criteria and the angiographic report had to be incorporated. Although this study was not randomized, it clearly reemphasizes the need for accurate preoperative viability imaging in patients with ischemic cardiomyopathy [36].

\section{Cardiovascular magnetic resonance imaging}

There were major advances in cardiovascular MRI (CMR) in 2010. Coronary CT angiography (CCTA) is usually preferred over CMR for diagnosing coronary artery disease. Scheffel et al. [37] looked at a series of 43 consecutive patients receiving both CCTA and perfusion CMR with late gadolinium enhancement and found that CCTA outperformed CMR for sensitivity and NPV [37]. CMR was more specific and had a higher PPV. These results suggest that CCTA is better suited for excluding CAD. 
A useful closed-chest animal model for chronic coronary artery stenosis was developed by $\mathrm{Wu}$ et al. and assessed with CMR [38]. Insights into the effects from chronic myocardial hypoperfusion may be gained from this model.

A 3-dimensional MR coronary angiography sequence was used for screening for coronary anomalies in 360 healthy people [39]. A malignant variant was identified in $1 \%$ of the subjects. Since $14 \%$ of sudden cardiac death in young athletes is caused by coronary anomalies, screening with CMR may avoid these tragic deaths.

Black blood sequences were proposed as a means to better understand peripheral vein bypass graft maturation and disease [40]. This may lead to improved diagnoses for treatment and repair.

CMR has been used to better understand atherosclerotic plaque vulnerability with carotid artery disease. Sadat et al. compared carotid plaque from 46 acutely symptomatic patients to 54 asymptomatic patients with moderate stenoses and found that the percent plaque lipid volume was lower for the symptomatic patients presumably due to escape of lipid during plaque rupture [41]. They suggest that plaque hemorrhage may be a better predictor. Serial CMR was performed in symptomatic and asymptomatic patients with recent intraplaque hemorrhage [42]. The degree of coronary artery stenosis was found to increase at 18 months more for the symptomatic patients and repeated plaque hemorrhage was more common. Another study showed that the shape and location of plaque calcification are important features associated with the presence of intraplaque hemorrhage [43].

There were a number of advances in reporting aspects of myocardial tissue characterization. Śpiewak et al. showed that the method for measuring the gray zone in late gadolinium enhanced images can provide different results for the amount of fibrosis in patients with hypertrophic cardiomyopathy [44]. Quantitative measures of first-pass myocardial perfusion and late gadolinium enhancement were found to be no better than visual inspection for predicting depressed ejection fraction or major adverse cardiac events following STEMI in a series of 192 patients [45]. Late gadolinium enhancement was recently found to be an important predictor of major adverse cardiac events in a series of 1,644 hypertensive patients with known or suspected coronary artery disease [46]. Reperfusion injury following percutaneous coronary intervention as manifest by intramyocardial hemorrhage was examined in relation to infarct size, microvascular obstruction and function in 45 patients [47]. Intramyocardial hemorrhage was not found to have prognostic significance beyond micovascular obstruction. Another study showed increased T2-weighted signal from affected myocardial segments of patients with left ventricular ballooning syndrome [48].

There were further advances relating to the measurement of ventricular mass and cardiovascular function. New allometric indices for left ventricular mass were developed by Brumback et al. to compare estimates of the prevalence and predictive value of left ventricular hypertrophy [49]. The prevalence of hypertrophy was higher for indices which did not account for weight. The predictive value of hypertrophy was significantly better with the new allometric height weight index than with un-indexed LV mass and may be better than indices without weight.

Intra-observer and interobserver variability of biventricular function, volumes and mass in patients with congenital heart disease (CHD) were reported by Luijnenburg et al. [50]. This is important since these measurements are frequently made for CHD patients and contribute to decision making and timing of (re)interventions.

The use of oblique 3D MRI tags was proposed by Shimuzu et al. [51]. The advantage of their technique is that it permits the tracking of tags even when they are dense to allow for estimation of 3D cardiac motion parameters.

An optimized method for measuring pixel-by-pixel flow velocity and shear stress from phase contrast MR images was proposed by Kim et al. [52]. The method was validated with a phantom and found to be more accurate than commercially available software. Related to this work, the accuracy and clinical utility of an automated method of image analysis of 4D magnetic resonance of the aorta was demonstrated by Johnson et al. [53].

\section{Computed tomography}

In a utilization analysis of cardiac care services over a four-year period (2004-2007), Karlsberg et al. [54] evaluated the impact of coronary $\mathrm{CT}$ angiography on 
downstream testing. Their data included electronic medical record and practice management review to capture treadmill stress testing, stress echocardiography, SPECT MPI, calcium scoring, coronary CTA, digital subtraction angiography (DSA), and percutaneous coronary intervention (PCI). Their findings strongly support appropriate implementation of coronary CTA into practice, with an overall reduction in the use of DSA and a significant increase in the fraction of catheter based angiography where intervention is performed. Although there were minor study limitations, the authors concluded that the high negative predictive value of CTA reduces unnecessary catheterizations, improves the triage patients, and saves health care money. In a similar analysis, Nielsen et al. [55] demonstrated that a frontline diagnostic use in symptomatic patients of exercisestress testing in comparison to CTA leads to more downstream diagnostic test utilization [55]. Future prospective trials are needed in order to define the most cost-effective diagnostic use of CTA relative to other diagnostic strategies.

As noted by Otero and colleagues [56] using the Tufts Medical Center cost-effectiveness analysis registry over a nine-year period (2000-2008), the cardiovascular imaging literature as a whole has grown substantially. However, the total number of studies that incorporate cardiovascular cost-utility analyses remains sparse. This is particularly true and problematic, for coronary CTA where there is a strong concern regarding overutilization, despite forward-thinking studies focused on the most efficient use of new technologies. However, studies including CT have, in general, favorable cost-effectiveness profiles with major determinants relating to being compared against observation, medical or no intervention instead of other imaging tests. In the context of the impact of imaging on clinical outcomes in comparative effectiveness research, Abdulla et al. described the prognostic value of absence or presence of coronary artery disease determined by CTA in a systematic review and meta-analysis [57].

The aggregate of 2010 CT articles included important data from a variety of CT technologies. Van der Bijl et al. [58] used $320 \times 0.5 \mathrm{~mm}$ detector CT, introduced 2 years earlier in our journal [59], to assess the impact of 0.5 versus $3.0 \mathrm{~mm}$ slice thickness reconstructions in calcium scoring. They found that $21 \%$ of patients who did not meet the CT threshold criteria for calcium on more traditional $3.0 \mathrm{~mm}$ slice reconstructions had coronary regions (minimum lesion area $>1 \mathrm{~mm}^{2}$ ) with attenuation $>130$ Hounsfield Units (HU). These findings emphasize the limitations with calcium scoring and the fact that a calcium score of zero does not exclude $\mathrm{CAD}$; in particular some patients may present with only non-calcified lesions. Plaque characterization in 35 patients with left anterior descending (LAD) lesions using 256-slice CT [60] was the focus of a study by Klass et al. [61]. Using semi-automated detection software, they reported lower inter-observer variability in the assessment of lesion volume when compared to calcified and complex (calcified plus non-calcified components) lesions. Chopard et al. [62] described the limitations of in vivo validation of CTA against an IVUS reference as $40 \mathrm{MHz}$ IVUS suffers from acoustic ambiguities in plaque characterization, and 64-slice MDCT is limited in analyzing plaque morphology and components.

There was also a variety of novel CT applications in the aggregate of 2010 articles. Yong et al. [63] retrospectively compiled a cardiovascular risk factor matched, non-obese (BMI $\left.<30 \mathrm{~kg} / \mathrm{m}^{2}\right) 200$ patient database (100 normal versus 100 abnormal) to study the relationship between pericardial fat and CAD as determined by CTA. Data shows that pericardial fat was significantly larger in those patients with CAD when compared to normal individuals and the findings persisted when those patients with BMI between 25 and $30 \mathrm{~kg} / \mathrm{m}^{2}$ (overweight but not obese) were excluded. Moreover, considering all 200 patients, the pericardial fat area correlated with atherosclerotic plaque score and CAD severity. Among the most novel of the application papers, van der Giessen et al. [64] combined coronary intravascular ultrasound (IVUS) and CT data in 23 patients (35 total arteries) to compute endothelial shear stress. The impact of shear stress on the endothelium, and in particular the role of low and very low shear stress states, leads to the development and progression of plaques, culminating in high-risk lesions that in turn can rupture and cause an acute coronary event [65]. This paper presents the threedimensional (3D) geometric model for the coronary lumen and wall using both modalities, capitalizing on the spatial resolution of IVUS and the volumetric spatial localization from CT angiography. The authors acknowledge that non-invasive (i.e. CT 
alone) wall shear stress calculations could be performed and are the subject of ongoing studies. However, the benefits of higher resolution from IVUS leads to robust input to computational models, at the expense of the risks and costs of IVUS that cannot translate to a screening procedure, which is a potential strength of this application.

The journal has continued to present novel approaches of image acquisition and reconstruction for radiation exposure. Bittencourt et al. [66] described an initial experience with iterative image reconstruction demonstrating reduced image noise and contrast-to-noise ratio in coronary $\mathrm{CT}$ angiography. Many future studies will contribute to the literature to translate the $\mathrm{CT}$ signal and contrast benefits to radiation reduction.

\section{Conflict of interest None}

Open Access This article is distributed under the terms of the Creative Commons Attribution Noncommercial License which permits any noncommercial use, distribution, and reproduction in any medium, provided the original author(s) and source are credited.

\section{References}

1. Janssen JP, Rares A, Tuinenburg JC, Koning G, Lansky AJ, Reiber JHC (2010) New approaches for the assessment of vessel sizes in quantitative (cardio)vascular X-ray analysis. Int J Cardiovasc Imaging 26:259-271

2. Tu S, Koning G, Jukema W, Reiber JHC (2010) Assessment of obstruction length and optimal viewing angle from biplane X-ray angiograms. Int $\mathrm{J}$ Cardiovasc Imaging 26:5-17

3. Garcia JA, Movassaghi B, Casserly IP et al (2010) Determination of optimal viewing regions for X-ray coronary angiography based on a quantitative analysis of 3D reconstructed models. Int $\mathbf{J}$ Cardiovasc Imaging 25:455-462

4. Liao R, Luc D, Sun Y, Kirchberg K (2010) 3-D reconstruction of the coronary artery tree from multiple views of a rotational X-ray angiography. Int $\mathbf{J}$ Cardiovasc Imaging 26:733-749

5. Hetterich H, Redel T, Lauritsch G, Rohkohl C, Rieber J (2010) New X-ray imaging modalities and their integration with intravascular imaging and interventions. Int J Cardiovasc Imaging 26:797-808

6. Van Tongeren RB, Hamming JF, le Cessie S, van Erkel AR, van Bockel JH (2010) Limited value of digital subtraction angiography in the evaluation of cell-based therapy in patients with limb ischemia. Int $\mathbf{J}$ Cardiovasc Imaging 26:19-25
7. Yuce M, Davutoglu V, Yavuz S et al (2010) Coronary sinus dilatation is associated with left ventricular systolic dysfunction and poor functional status in subjects with chronic heart failure. Int J Cardiovasc Imaging 26:541-545

8. Chockalingam A, Xie G-Y, Dellsperger KC (2010) Echocardiography in stress cardiomyopathy and acute LVOT obstruction. Int J Cardiovasc Imaging 26:527-535

9. Van der Wall EE, Holman ER, Scholte AJ, Bax JJ (2010) Echocardiography in takotsubo cardiomyopathy; a useful approach? Int J Cardiovasc Imaging 26:537-540

10. Van der Wall EE, Schalij MJ (2010) Mitral valve prolapse: a source of arrhythmias? Int J Cardiovasc Imaging 26: 147-149

11. Turker Y, Ozdayin M, Acar G et al (2010) Predictors of ventricular arrhythmias in patients with mitral valve prolapse. Int J Cardiovasc Imaging 26:139-145

12. Bilge A, Altigan D, Onur I et al (2010) Relationship between left ventricular hypertrophy, hypertensive retinopathy, microalbuminuria and echocardiographic modalities in newly diagnosed hypertensive patients. Int $\mathbf{J}$ Cardiovasc Imaging 26:405-412

13. Aypar E, Alehan D, Hazirolan T, Gumruk F (2010) The efficacy of tissue Doppler imaging in predicting myocardial iron load in patients with beta-thalassemia major: correlation with $\mathrm{T} 2 *$ cardiovascular magnetic resonance. Int J Cardiovasc Imaging 26:413-421

14. Van der Wall EE, Bax JJ, Jukema JW, Schalij MJ (2010) Distal protection beneficial? Int J Cardiovasc Imaging 26:135-138

15. Dyan Y-Y, Zhang H-B, Liu L-W et al (2010) Effects of distal protection on left ventricular function in acute anterior myocardial infarction: a Doppler echocardiographic study. Int J Cardiovasc Imaging 26:125-133

16. Pavlopoulos H, Nihoyannopoulos P (2010) Recent advances in cardiac resynchronization therapy: echocardiographic modalities, patient selection, optimization, nonresponders-all you need to know for more efficient CRT. Int J Cardiovasc Imaging 26:177-191

17. Baur LH (2010) Echocardiography as a guidance in CRT management: the GPS system in a labyrinth? Int J Cardiovasc Imaging 26:193-195

18. Ozdemir A, Kaya C, Ozcan O et al (2010) Prediction of subclinical left ventricular dysfunction with longitudinal two-dimensional strain and strain rate imaging in patients with mitral stenosis. Int J Cardiovasc Imaging 26:397-404

19. Valocik G, Druzbacka L, Valocikova I, Mitro P (2010) Velocity vector imaging to quantify left atrial function. Int J Cardiovasc Imaging 26:641-649

20. Li CH, Carreras F, Leta R, Carballeira L, Pujadas S, PonsLlado G (2010) Mechanical left ventricular dyssynchrony detection by endocardium displacement analysis with 3D speckle tracking technology. Int $\mathrm{J}$ Cardiovasc Imaging 26:867-870

21. Innocenti F, Agresti C, Baroncini C et al (2010) Prognostic value of dobutamine stress echocardiography in diabetic patients. Int J Cardiovasc Imaging 26:499-507

22. Anwar AM, Nosir YFM, Ajam A, Chamsi-Pasha H (2010) Central role of three dimensional echocardiography in the assessment of intracardiac thrombi. Int $\mathrm{J}$ Cardiovasc Imaging 26:519-526 
23. Xue H, Sun K, Yu J et al (2010) Three dimensional echocardiographic virtual endoscopy for the diagnosis of congenital heart disease in children. Int $\mathrm{J}$ Cardiovasc Imaging 26:851-859

24. Paul B, Minocha A (2010) Thrombosis of a bileaflet prosthetic mitral valve: a real-time three-dimensional transeosophageal echocardiography perrspective. Int $\mathrm{J}$ Cardiovasc Imaging 26:367-380

25. Gripari P, Tamborini G, Barbier P et al (2010) Real-time three-dimensional transoesophageal echocardiography: a new intraoperative feasible and useful technology in cardiac surgery. Int J Cardiovasc Imaging 26:651-660

26. Zhu H, Zhang W, Zhong M, Zhang G, Zhang Y (2010) Myocardial ultrasonic integrated backscatter analysis in patients with chronic atrial fibrillation. Int $\mathrm{J}$ Cardiovasc Imaging 26:861-865

27. Davies JR, Izquierdo-Garcia D, Rudd J et al (2010) FDGPET can distinguish inflamed from non-inflamed plaque in an animal model of atherosclerosis. Int $\mathrm{J}$ Cardiovasc Imaging 26:41-48

28. Van der Wall EE, Schalij MJ, van der Laarse A, Bax JJ (2010) Cardiac resynchronization therapy; the importance of evaluating cardiac metabolism. Int J Cardiovasc Imaging 26:293-297

29. Kitaizumi K, Yukiiri K, Masugata H et al (2010) Acute improvement of cardiac efficiency measured by $11 \mathrm{C}$-acetate PET after cardiac resynchronization therapy and clinical outcome. Int J Cardiovasc Imaging 26:285-292

30. Van der Wall EE, Scholte AJ, Bax JJ (2010) Gated myocardial SPECT imaging: true additional value in AMI? Int J Cardiovasc Imaging 26:893-896

31. Neill J, Harbinson M, Adgey J (2010) Myocardial wall motion and thickening assessment in early gated SPECT images of acute coronary syndrome patients likely to have inferolateral perfusion defects. Int $\mathrm{J}$ Cardiovasc Imaging 26:881-891

32. Muzzarelli S, Pfisterer ME, Brand JM, Zellweger MJ (2010) Gate-keeper to coronary angiography: comparison of exercise testing, myocardial perfusion SPECT and individually tailored approach for risk stratification. Int $\mathbf{J}$ Cardiovasc Imaging 26:871-879

33. Forster S, Rieber J, Ubleis C et al (2010) Tc-99m sestamibi single photon emission computed tomography for guiding percutaneous coronary intervention in patients with multivessel disease: a comparision with quantitative coronary angiography and fractional flow reserve. Int $\mathrm{J}$ Cardiovasc Imaging 26:203-213

34. Rees M (2010) The relationship between myocardial SPECT and fractional flow reserve: is it drifting apart? Int $\mathbf{J}$ Cardiovasc Imaging 26:215-216

35. Boehm J, Haas F, Bauernschmitt R et al (2010) Impact of preoperative positron emission tomography in patients with severely impaired LV function undergoing surgical revascularization. Int J Cardiovasc Imaging 26:423-432

36. Van der Wall EE, Siebelink HM, Scholte AJ, Bax JJ (2010) Positron emission tomography: viable tool in patients preCABG? Int J Cardiovasc Imaging 26:661-664

37. Scheffel H, Stolzmann P, Alkadhi H et al (2010) Low-dose CT and cardiac MR for the diagnosis of coronary artery disease: accuracy of single and combined approaches. Int J Cardiovasc Imaging 26:579-590
38. Wu M, Bogaert J, D'Hooge J et al (2010) Closed-chest animal model of chronic coronary artery stenosis. Assessment with magnetic resonance imaging. Int $\mathrm{J}$ Cardiovasc Imaging 26:299-308

39. Prakken NH, Cramer MJ, Olimulder MA, Agostoni P, Mali WP, Velthuis BK (2010) Screening for proximal coronary artery anomalies with 3-dimensional MR coronary angiography. Int J Cardiovasc Imaging 26:701-710

40. Rybicki FJ, Mitsouras D, Owens CD et al (2010) Multicontrast high spatial resolution black blood inner volume three-dimensional fast spin echo MR imaging in peripheral vein bypass grafts. Int J Cardiovasc Imaging 26:683-691

41. Sadat U, Teng Z, Young VE, Graves MJ, Gillard JH (2010) Three-dimensional volumetric analysis of atherosclerotic plaques: a magnetic resonance imaging-based study of patients with moderate stenosis carotid artery disease. Int $\mathbf{J}$ Cardiovasc Imaging 26:897-904

42. Wang Q, Wang Y, Cai J, Cai Y, Ma L, Xu X (2010) Differences of signal evolution of intraplaque hemorrhage and associated stenosis between symptomatic and asymptomatic atherosclerotic carotid arteries: an in vivo highresolution magnetic resonance imaging follow-up study. Int J Cardiovasc Imaging 26:323-332

43. Xu X, Ju H, Cai J, Cai Y, Wang X, Wang Q (2010) Highresolution MR study of the relationship between superficial calcification and the stability of carotid atherosclerotic plaque. Int J Cardiovasc Imaging 26(Suppl 1):143-150

44. Spiewak M, Malek LA, Chojnowska L et al (2010) Late gadolinium enhancement gray zone in patients with hypertrophic cardiomyopathy. Comparison of different gray zone definitions. Int $\mathbf{J}$ Cardiovasc Imaging 26:693-699

45. Husser O, Bodi V, Sanchis J et al (2010) Head to head comparison of quantitative versus visual analysis of contrast CMR in the setting of myocardial stunning after STEMI: implications on late systolic function and patient outcome. Int J Cardiovasc Imaging 26:559-569

46. Krittayaphong R, Boonyasirinant $\mathrm{T}$, Chaithiraphan V (2010) Prognostic value of late gadolinium enhancement in hypertensive patients with known or suspected coronary artery disease. Int $\mathrm{J}$ Cardiovasc Imaging 26(Suppl 1): 123-131

47. Beek AM, Nijveldt R, van Rossum AC (2010) Intramyocardial hemorrhage and microvascular obstruction after primary percutaneous coronary intervention. Int J Cardiovasc Imaging 26:49-55

48. Joshi SB, Chao T, Herzka DA et al (2010) Cardiovascular magnetic resonance T2 signal abnormalities in left ventricular ballooning syndrome. Int $\mathbf{J}$ Cardiovasc Imaging 26:227-232

49. Brumback LC, Kronmal R, Heckbert SR et al (2010) Body size adjustments for left ventricular mass by cardiovascular magnetic resonance and their impact on left ventricular hypertrophy classification. Int $\mathrm{J}$ Cardiovasc Imaging 26:459-468

50. Luijnenburg SE, Robbers-Visser D, Moelker A, Vliegen HW, Mulder BJ, Helbing WA (2010) Intra-observer and interobserver variability of biventricular function, volumes and mass in patients with congenital heart disease measured by $\mathrm{CMR}$ imaging. Int $\mathbf{J}$ Cardiovasc Imaging 26:57-64 
51. Shimizu Y, Amano A, Matsuda T (2010) Oblique 3D MRI tags for the estimation of true $3 \mathrm{D}$ cardiac motion parameters. Int J Cardiovasc Imaging 26:905-921

52. Kim T, Seo JH, Bang SS, Choi HW, Chang Y, Lee J (2010) Optimization of MR phase-contrast-based flow velocimetry and shear stress measurements. Int $\mathbf{J}$ Cardiovasc Imaging 26(Suppl 1):133-142

53. Johnson RK, Premraj S, Patel SS et al (2010) Automated analysis of four-dimensional magnetic resonance images of the human aorta. Int J Cardiovasc Imaging 26:571-578

54. Karlsberg RP, Budoff MJ, Thomson LEJ, Friedman JD, Berman DS (2010) Reduction in downstream test utilization following introduction of coronary computed tomography in a cardiology practice. Int J Cardiovasc Imaging 26:359-366

55. Nielsen LH, Markenvard J, Jensen JM, Mickley H et al (2011) Frontline diagnostic evaluation of patients suspected of angina by coronary computed tomography reduces downstream resource utilization when compared to conventional ischemia testing. Int $\mathrm{J}$ Cardiovasc Imaging 27. doi:10.1007/s10554-010-9737-6

56. Otero HJ, Rybicki FJ, Greenberg D, Mitsouras D, Mendoza JA, Neumann PJ (2010) Cost-effective diagnostic cardiovascular imaging: when does it provide good value for the money? Int J Cardiovasc Imaging 26:605-612

57. Abdulla J, Asferg C, Kofoed KF (2011) Prognostic value of absence or presence of coronary artery disease determined by 64-slice computed tomography coronary angiography: a systematic review and meta-analysis. Int $\mathbf{J}$ Cardiovasc Imaging 27: [Epub ahead of print]

58. Van der Bijl N, de Bruin PW, Geleijns J et al (2010) Assessment of coronary artery calcium by using volumetric 320-row multi-detector computed tomography: comparison of $0.5 \mathrm{~mm}$ with $3.0 \mathrm{~mm}$ slice reconstructions. Int J Cardiovasc Imaging 26:473-482
59. Rybicki FJ, Otero HJ, Steigner ML et al (2008) Initial evaluation of coronary images from 320-detector row computed tomography. Int $\mathrm{J}$ Cardiovasc Imaging 24: 535-546

60. Klass O, Kleinhans S, Walker MJ et al (2010) Coronary plaque imaging with 256-slice multidetector computed tomography: interobserver variability of volumetric lesion parameters with semiautomatic plaque analysis software. Int J Cardiovasc Imaging 26:711-720

61. Walker MJ, Olszewski ME, Desai MY, Halliburton SS, Flamm SD (2010) New radiation dose saving technologies for 256-slice cardiac computed tomography angiography. Int J Cardiovasc Imaging 25:189-199

62. Wann S (2010) Characterization of coronary arterial plaque. Int J Cardiovasc Imaging 26:385-386

63. Yong HS, Kim EJ, Seo HS et al (2010) Pericardial fat is more abundant in patients with coronary atherosclerosis and even in the non-obese patients: evaluation with cardiac CT angiography. Int J Cardiovasc Imaging 26:53-62

64. Van der Giessen AG, Schaap M, Gijsen FJH et al (2010) 3D fusion of intravascular ultrasound and coronary computed tomography for in vivo wall shear stress analysis. Int J Cardiovasc Imaging 26:781-796

65. Rybicki FJ, Melchionna S, Mitsouras D et al (2009) Prediction of coronary artery plaque progression and potential rupture from 320-detector row prospectively ECG-gated single heart beat CT angiography: Lattice Boltzmann evaluation of endothelial shear stress. Int $\mathrm{J}$ Cardiovasc Imaging 25:289-299

66. Bittencourt MS, Schmidt B, Seltmann M et al (2011) Iterative reconstruction in image space (IRIS) in cardiac computed tomography: initial experience. Int J Cardiovasc Imaging 27. doi:10.1007/s10554-010-9756-3 